\title{
November 2018 Arizona Thoracic Society Notes
}

The November 2018 Arizona Thoracic Society meeting was held on Wednesday, November 28, 2018 at the HonorHealth Rehabilitation Hospital beginning at 6:30 PM. This was a dinner meeting with case presentations. There were 10 in attendance representing the pulmonary, critical care, sleep, and radiology communities.

At the beginning of the meeting the Tobacco 21 bill was discussed. This bill has been twice held up in the Arizona House of Representatives Commerce Committee by the committee chairman. It was noted that one of the healthcare executives had financially supported the reelection campaign of the commerce committee chairman. A decision was made to send a letter to the healthcare executive pointing the pro-tobacco stance of the committee chairman. CME for the Arizona Thoracic Society meetings was also discussed. CME case presentations that had been previously approved will be presented and CME granted through the University of Arizona.

There were 3 case presentations:

1. Dr. Richard Robbins presented for Dr. Robert Raschke a case of a 54-year-old man with neck pain that eventually proved to be a paraspinous abscess.

2. Dr. Mike Gotway presented a 36-year-old woman who presented with respiratory failure. She eventually proved to have been self-injecting hydrocodone.

3. Dr. Lewis Wesselius presented a 28-year-old man from Tennessee with enlarging lung nodules who had been treated for presumed histoplasmosis, but was having hemoptysis and clinically worsening. Lung biopsy demonstrated metastatic angiosarcoma.

The meeting was adjourned about 8:30 PM. The next meeting will be on January 23, 2019 at 6:30 PM at HonorHealth Rehabilitation Hospital. 